\title{
Intention to Implement a Healthy Lifestyle: Does Acculturation Make a Difference?
}

\author{
Deshields S1, Joachim Celestin M11, Lara M1, Miller LM11, DaCosta \\ Davis SS ${ }^{1}$, Montgomery S ${ }^{1}$ and Clarke $\mathrm{C}^{2 *}$ \\ ${ }^{1}$ Loma Linda University, USA \\ ${ }^{2}$ Department of Medicine and Behavioral Health, Loma Linda University, USA
}

Research Article

Volume 2 Issue 6

Received Date: June 18, 2019

Published Date: November 13, 2019

DOI: $10.23880 /$ jqhe-16000144

*Corresponding author: Camille Clarke, MD, MBA, FACP, Department of Medicine and Behavioral Health, School of Medicine and Behavioral Health, Loma Linda University, 11065 Campus Street, Loma Linda CA 92350, USA, Tel: (909) 651-5881; Email: cclarke@llu.edu

\section{Abstract}

Studies have examined the relationship between acculturation and a healthy lifestyle within a representative population of Latinos. However, few studies describe the impact of acculturation on Latino individuals' intent to adopt a healthier lifestyle and diet. The purpose of this study was to examine the association between acculturation and intent to change one's diet and exercise habits to achieve a healthier lifestyle. In a survey administered to 50 Latinos in Perris, California we found that US birth was significantly associated with increased intent to eat healthy $(p<.01)$ and intent to exercise $(p<.05)$, suggesting the need to address acculturation when considering lifestyle recommendations.

Keywords: Acculturation; Latinos; Health Promotion; Obesity

\section{Introduction}

Among minority groups, Latinos have one of the highest prevalence of obesity, a known risk factor for lifestyle-based diseases including diabetes, heart disease, and cancer [1]. To address obesity in this population it is important to understand the factors that influence individuals' willingness to initiate a healthy lifestyle. Our study sought to examine how varying degrees of acculturation for Latinos within the United States, as measured by language preference, is associated with respondent's intent to adopt a healthy lifestyle.

\section{Methods}

Our study used a community-based participatory research approach. For the quantitative data collection, pre- and post-assessment surveys were developed with input from community members, measuring participants' demographics, health, lifestyle behaviors, family history, impact of religion on personal health, self-efficacy, and intent to change. Before use in a lifestyle program for lowincome immigrant Latinos, surveys were piloted $(\mathrm{N}=50)$ with bilingual and monolingual Latinos 18 years and older.

Specific variables including demographics, lifestyle, and social history in the pilot survey were collected to assess correlations between acculturation and intent to change. As a proxy for acculturation, participants were asked to indicate their country of birth, the language(s) read and spoken primarily, and language(s) they speak most frequently at home and while conversing with friends. Participant's intent to change was measured by questions asking their intent to exercise and to eat healthy. SPSS v. 25 was used to identify correlations 


\section{Journal of Quality in Health Care \& Economics}

between acculturation and intent to engage in healthy lifestyle practices and exercise.

\section{Results}

Demographics of study participants are summarized in Table 1 below. The mean age of participants was 45.5 $( \pm 16.91)$ years of age and the majority $(70 \%)$ of participants were female. Only $30 \%$ of survey respondents were born in the United States, while the majority came from Mexico and South America. The majority of respondents also indicated that they read and spoke mostly in Spanish. Statistical analyses were conducted by using one-way ANOVA to compare the relationship between acculturation and intent to engage in healthy lifestyle practices. Our findings showed that English language preference was positively correlated (Spearman's rho $=.371)$ with intent to eat healthy $(\mathrm{p}<.01)$, and positively correlated (Spearman's rho $=.356$ ) with intent to exercise ( $\mathrm{p}<.05)$, as shown below in Figures 1-3. Ad hoc analyses revealed that those who only spoke and read English had more intention of exercising $(+0.69$, $p=.000)$ and of eating healthy $(+0.66, p=.01)$ than those who spoke and read mainly Spanish (Figure 3).

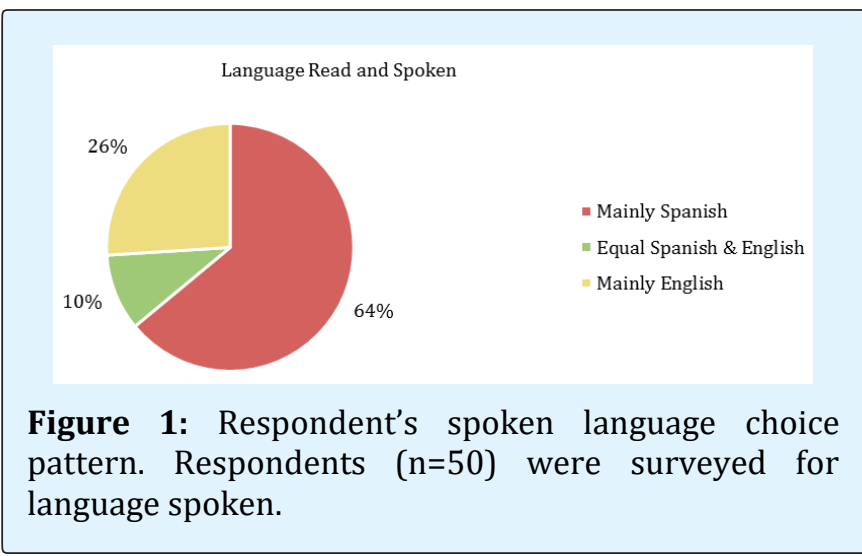

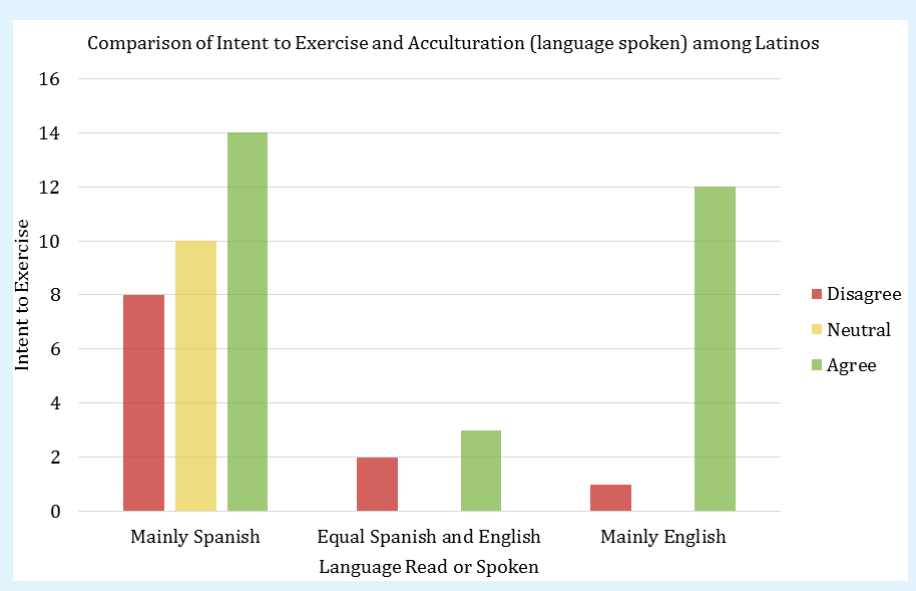

Figure 2: Distribution of language patterns by intent to exercise According to Spearman's rho, language preference (a proxy for acculturation, in this case preference for English) is positively correlated (.356*) with intent to exercise $\mathrm{p}<.05^{*}$.
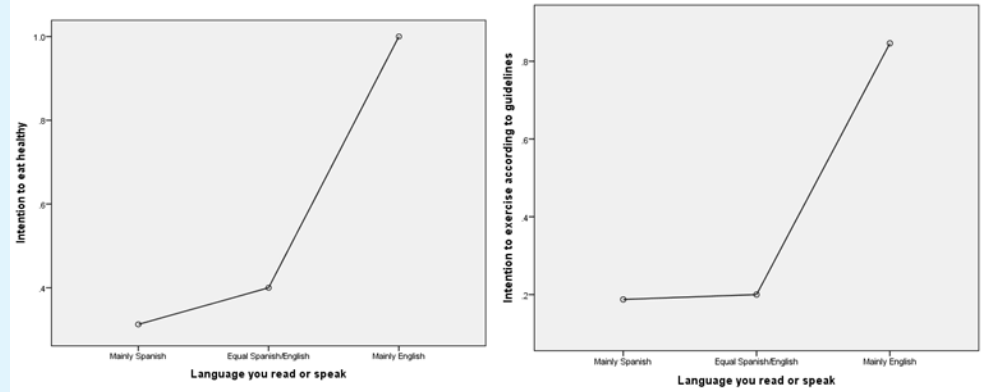

Figure 3: Respondent's language patterns and healthy eating and exercise intentions. Ad hoc analyses revealed that those who spoke and read English had more intention of exercising $(+0.69, p=.000)$ and of eating healthy $(+0.66$, $p=.01$ ) compared to those who only spoke and read mainly Spanish. 


\section{Journal of Quality in Health Care \& Economics}

\section{Discussion}

Results from our study with low income Latino adults (mostly middle-aged) show that higher acculturation, as demonstrated by the preferential use of English for both speaking and reading, was associated with increased intent to eat healthy and exercise. These findings are consistent with previous studies that indicate that increased acculturation is associated with increased likelihood of engaging in healthy behaviors [1].

On the contrary, other studies among Latino adolescents suggest that higher levels of acculturation were associated with a decreased likelihood of exhibiting health-promoting behaviors such as having adequate vitamin intake, eating breakfast, and sleeping adequate hours of sleep at night [2]. Still, Latinos are also less likely to engage/succeed in lifestyle and weightless programs [3-10]. Studies on the impact of acculturation and obesity show an increased obesity risk associated with length of residence for Latino immigrants [3]. While recent Latino immigrants tend to have a healthier diet than U.S. born Latinos, this distinction declines over time (Tables $1 \& 2$ ). The shift away from healthier lifestyle practices as length of residence in the US increases likely reflects changes in physical activity and dietary practices: less physical activity and increased consumption of unhealthy foods [4]. Several research studies report that increased acculturation to the US is associated with a reduction in healthy components of the Latino diet - for instance fiber [5] as well as fruit, rice, and beans and an increased consumption of sugar and sweetened beverages [6].

\begin{tabular}{|c|c|c|}
\hline & Mean ( \pm SD) & N (\%) \\
\hline Age (years) & $45.46( \pm 16.91)$ & \\
\hline Gender & & $15(30 \%)$ \\
\hline Male & & $35(70 \%)$ \\
\hline Female & & $35(70 \%)$ \\
\hline Country of origin & & $15(30 \%)$ \\
\hline Mexico/So. America & & \\
\hline USA & & $36(72 \%)$ \\
\hline Language read or spoken & & $5(10 \%)$ \\
\hline Mainly Spanish & & $13(26 \%)$ \\
\hline Equal English/Spanish & & $36(72 \%)$ \\
\hline Mainly English & & $4(8 \%)$ \\
\hline Language spoken at home & $10(20 \%)$ \\
\hline Mainly Spanish & & \\
\hline Equal English/Spanish & $33(66 \%)$ \\
\hline Mainly English & & $5(10 \%)$ \\
\hline Language spoken with friends & & $12(24 \%)$ \\
\hline Mainly Spanish & & \\
\hline Equal English/Spanish & & \\
\hline Mainly English & & \\
\hline
\end{tabular}

Table 1: Baseline demographics of study Respondents.

\begin{tabular}{|c|c|c|c|}
\hline & & Intention to exercise & Intention to eat healthy \\
\hline \multirow{3}{*}{ Language read \& spoken } & Spearman correlation & $0.37^{*}$ & $0.36^{*}$ \\
\cline { 2 - 4 } & Sig. (2-tailed) & 0.008 & 0.01 \\
\cline { 2 - 4 } & $\mathrm{N}=$ & 50 & 50 \\
\hline \multirow{3}{*}{ Language spoken at home } & Spearman correlation & $0.33^{*}$ & 0.2 \\
\cline { 2 - 4 } & Sig. (2-tailed) & 0.02 & 0.16 \\
\cline { 2 - 4 } & $\mathrm{N}=$ & 50 & 50 \\
\hline \multirow{3}{*}{ Language spoken with friends } & Spearman correlation & $0.40^{*}$ & $.32^{*}$ \\
\cline { 2 - 4 } & Sig. (2-tailed) & 0.004 & 0.02 \\
\cline { 2 - 4 } & $\mathrm{N}=$ & 50 & 0.5 \\
\hline
\end{tabular}

*Correlation is significant at the 0.01 level (2-tailed).

Table 2: Correlation between language used most frequently and intention to exercise and eat healthy. 


\section{Journal of Quality in Health Care \& Economics}

Overall the respondents in our survey were older and less acculturated than the broader Latino population in Southern California which may well have contributed to their lack of intent to improve their dietary practices or exercise (vs. those respondents who were more likely to speak and read English i.e., those more acculturated) as they may already (and still) have healthier eating habits. However, over time, as individuals live longer in the US, the protective diet seems to gradually disappear and, together with a lack of intent to change behavior, increases their risk of becoming overweight/obese. In turn, these new behaviors and changes in intent and body mass index result in an increased risk of many lifestylerelated diseases such as diabetes, cardiovascular diseases and some cancers [3]. Given the overall high rate of obesity in Latinos, and such differences between subgroups of Latinos based on their degree of acculturation, there is a clear need for culturally targeted lifestyle programs that take into account openness to engage in health behaviors, especially among vulnerable groups such as more recent immigrants.

Our data suggest that lifestyle programs need to find a way to more effectively motivate recent immigrants to retain their protective health behaviors before they lose them. In addition, stressors that may contribute to a reluctance to participate in health promotion programs $[4,5]$, such as limited access to healthcare, discrimination, legal status, and challenges associated with communicating in a new language should be acknowledged and mitigated. Our findings confirm that Latinos are not a monolithic linguistically homogenous group and point to a need for programs targeting less acculturated Latinos as their readiness, needs and challenges may differ from the more acculturated ones.

\section{Conclusion}

- In the United States, Latinos make up the largest minority and have the highest prevalence of obesity.

- While a balanced diet is essential for a healthy lifestyle, it is heavily influenced by cultural background and ethnicity.

- The findings from our study suggest openness for positive lifestyle changes in more acculturated Latinos.

- However, the need remains for increased and distinctive programming for newly migrated individuals.

- Interventions targeted towards differing Latinos groups may provide a critical opening for obesityprevention interventions.

- Health educators and healthcare providers working with Latinos would benefit from assessing

$$
\begin{aligned}
& \text { acculturation prior to making lifestyle } \\
& \text { recommendations. }
\end{aligned}
$$

\section{References}

1. Hales CM, Margaret D, Carroll MSPH, Cheryl D, Fryar, et al. (2017) Prevalence of Obesity Among Adults and Youth: United States, 2015-2016. Centers for Disease Control and Prevention.

2. Pérez Escamilla R (2011) Acculturation, nutrition, and health disparities in Latinos. Am J Clin Nutr 93(5): 1163-1167.

3. Kaplan MS, Huguet N, Newsom JT, McFarland BH (2004) The association between length of residence and obesity among Hispanic immigrants. American Journal of Preventive Medicine 27(4): 323-326.

4. Mikolajczyk RT, Bredehorst M, Khelaifat N, Maier C, Maxwell AE (2007) Correlates of depressive symptoms among Latino and Non-Latino White adolescents: Findings from the 2003 California Health Interview Survey. BMC Public Health 7: 21.

5. Ayala GX, Baquero B, Klinger S (2008) A systematic review of the relationship between acculturation and diet among Latinos in the United States: implications for future research. J Am Diet Assoc 108(8): 13301044.

6. Hammelgreen D, Daza NR, Copper E, Martinez D (2007) "I Don't Make the Soups Anymore": Pre- to Post-Migration Dietary and Lifestyle Changes Among Latinos Living in West-Central Florida. Taylor \& Francis pp: 427-444.

7. Crespo CJ, Smit E, Carter Pokras O, Andersen R (2001) Acculturation and leisure-time physical inactivity in Mexican American adults: results from NHANES III, 1988-1994. Am J Public Health 91(8): 1254-1257.

8. Venditti EM, Wylie Rosett J, Delahanty LM, Mele L, Hoskin MA, et al. (2014) Short and long-term lifestyle coaching approaches used to address diverse participant barriers to weight loss and physical activity adherence. Springer Link 11: 16.

9. Jackson L (2009) Translating the Diabetes Prevention Program into practice: a review of community interventions. The Diabetes Educ 35(2): 309-320.

10. Rosas LG, Thiyagarajan S, Goldstein BA, Drieling RL, Romero PP, et al. (2015) The effectiveness of two community-based weight loss strategies among obese, low-income US Latinos. J Acad Nutr Diet 115(4): 537-550. 